\section{Prevalência e fatores associados ao sobrepeso e à obesidade em adolescentes, estudantes de escolas de Ensino Médio de Pernambuco, Brasil}

\author{
Prevalence of overweight and obesity and \\ associated factors among public high \\ school students in Pernambuco \\ State, Brazil
}

Rafael Miranda Tassitano 1,2,3,4 Mauro Virgilio Gomes de Barros 1,2 Maria Cecilia Marinho Tenório 1,2 Jorge Bezerra 1,2,4 Pedro C. Hallal 5,6

\author{
1 Programa de Pós-graduação \\ em Hebiatria, Universidade \\ de Pernambuco, Recife, Brasil. \\ 2 Programa de Pós-graduação \\ em Educação Física, \\ Universidade de Pernambuco, \\ Recife, Brasil. \\ ${ }^{3}$ Departamento de Educação \\ Física Universidade Federal \\ Rural de Pernambuco, Recife, \\ Brasil. \\ ${ }_{4}^{4}$ Associação Caruaruense de \\ Ensino Superior, Caruaru \\ Brasil. \\ 5 Programa de Pós- \\ graduaç̃o em Epidemiologia \\ Universidade Federal de \\ Pelotas, Pelotas, Brasil. \\ ${ }_{6}$ Programa de Pós-graduação \\ em Educação Física, \\ Universidade Federal de \\ Pelotas, Pelotas, Brasil. \\ Correspondência \\ R. M. Tassitano \\ Programa de Pós-graduação \\ em Hebiatria, Universidade \\ de Pernambuco. \\ Rua Teles Júnior 33, \\ apto. 501, Recife, $P E$ \\ 52050-040, Brasil. \\ rafael.tassitano@gmail.com
}

\section{Abstract}

This study aims to analyze the association between demographic, socioeconomic, school-related, and behavioral factors and overweight and obesity in adolescents. The sample included 4,210 public high school students (14-19 years old) in Pernambuco State, Brazil, selected by two-stage cluster sampling. Obesity and overweight were assessed using anthropometric measurements, and the Global School-Based Health Survey was used to collect personal and behavioral data. The cutoff points for defining overweight and obesity were those recommended by the International Obesity Task Force. Prevalence rates for overweight and obesity were 11.5\% (95\%CI: 10.7-12.8) and 2.4\% (95\%CI: 1.9-2.9), respectively. Overweight and obesity were more common in males who reported residing in urban areas and were not enrolled in physical education classes. Watching television 3+ hours/day was associated with obesity in females. Reducing TV time and encouraging enrollment in physical education could be effective measures to counteract the growing obesity trends.

Obesity; Overweight; Adolescent Health

\section{Introdução}

A obesidade alcançou proporções epidêmicas tanto em países desenvolvidos quanto em países em desenvolvimento, independente da faixa etária e sexo 1 . Mais recentemente, especialistas têm chamado a atenção para o aumento da prevalência de obesidade na infância e na adolescência 2,3. Esta preocupação decorre das comorbidades associadas (hipertensão, diabetes tipo 2 e problemas respiratórios) 2 e do impacto econômico e social 4 do aumento na prevalência de obesidade na infância e na juventude. Mas há, também, destaque para este problema em virtude das evidências de que a ocorrência na infância aumenta a chance de obesidade na vida adulta 5 .

Estudo conduzido por Wang et al. ${ }^{6}$ analisou a tendência temporal da prevalência de obesidade por intermédio de inquéritos nacionais em quatro países. Observou-se um aumento em três dos quatro países, sendo de $4,1 \%$ para $13,9 \%$ no Brasil (1975-1997), de 15,4\% para 25,6\% no Estados Unidos (1971-1974 e 1988-1994) e de 6,4\% para $7,7 \%$ na China (1991-1997), mas diminuiu de $15,6 \%$ para 9\% na Rússia (1992-1998). Estudo analisando dados relativos à América Latina também evidenciou um considerável aumento na proporção de crianças e os adolescentes obesos, especialmente nos últimos anos 7 .

No Brasil, estudos com representatividade nacional ou regional foram baseados em inquéri- 
tos nacionais realizados pelo Instituto Brasileiro de Geografia e Estatística (IBGE): Estudo Nacional de Despesa Familiar (ENDEF), Pesquisa Nacional sobre Saúde e Nutrição (PNSN), Pesquisa de Padrões de Vida (PPV) e Pesquisa de Orçamentos Familiares (POF), realizadas em 1975, 1989, 1999 e 2002-2003, respectivamente 8,9,10,11. De uma maneira geral, esses levantamentos evidenciaram que a prevalência de excesso de peso em crianças e adolescentes é maior nas regiões Sul e Sudeste e menor na Região Nordeste.

Na Região Nordeste como um todo e em Pernambuco, os estudos realizados foram com populações muito específicas, tais como: recrutas do Exército 12, moradores de favelas 13 e estudantes de uma ou duas escolas 14,15,16. De uma maneira geral, as amostras também foram muito heterogêneas em relação à idade (abrangendo simultaneamente tanto crianças quanto adolescentes) 13,15,16. Além disso, a validade interna e externa dos achados ficou limitada em razão do delineamento adotado, delimitação da população alvo do estudo ou em decorrência do modo de seleção dos sujeitos. Destaque-se, também, que nenhum levantamento com representatividade estadual foi localizado.

Fatores genéticos, fisiológicos, metabólicos, ambientais e comportamentais têm sido freqüentemente associados a esta condição 17,18. Identificar os fatores associados à ocorrência da obesidade é importante porque pode auxiliar o planejamento de intervenções.

Apesar de se dispor de razoável conhecimento sobre a prevalência de obesidade em diferentes grupos populacionais, há ainda relativa escassez de estudos sobre fatores associados à ocorrência do excesso de peso em adolescentes do Nordeste do Brasil. Em virtude disso, o objetivo deste estudo foi analisar a associação entre fatores demográficos, sócio-econômicos, escolares e comportamentais com a prevalência de sobrepeso e obesidade em adolescentes estudantes do Ensino Médio do Estado de Pernambuco.

\section{Métodos}

O presente estudo epidemiológico transversal foi desenvolvido como parte do projeto Estilos de Vida e Comportamento de Risco à Saúde do Estudante do Ensino Médio de Pernambuco: do Diagnóstico à Intervenção. O protocolo do estudo foi submetido ao Comitê de Ética em Pesquisa com Seres Humanos do Hospital Agamenon Magalhães, na cidade do Recife, Pernambuco, em atendimento à indicação do Sistema Nacional de Ética na Pesquisa com Seres Humanos, tendo sido aprovado em julho de 2005.
A população alvo foi limitada aos estudantes da rede pública estadual de Ensino Médio do Estado de Pernambuco, com faixa de idade entre 14 e 19 anos. Segundo dados fornecidos pela Secretaria de Educação do Estado, considerando-se todas as dependências administrativas (federal, estadual, municipal e privada), os sujeitos matriculados na rede pública estadual representam cerca de $80 \%$ do total de estudantes do Ensino Médio em Pernambuco. O dimensionamento da amostra foi efetuado de forma a atender os diversos objetivos do projeto, que incluíam a avaliação da exposição a dez fatores de risco à saúde, dentre os quais o sobrepeso e a obesidade.

Para o cálculo do tamanho da amostra foram utilizados os seguintes critérios: população estimada em 353 mil sujeitos; intervalo de 95\% de confiança (IC95\%); erro amostral de 3 pontos percentuais; prevalência estimada em 50\%; e, efeito de delineamento amostral estabelecido em quatro vezes o tamanho mínimo da amostra. Isso representaria uma amostra mínima com 4.217 sujeitos. Considerando o componente analítico do estudo, calculou-se que o tamanho amostral permitiria detectar como significativas odds ratio $(\mathrm{OR})$ superiores a 1,2 , considerandose poder estatístico de $80 \%$ e IC95\%.

Procurou-se garantir que a amostra selecionada representasse a população alvo quanto à distribuição conforme região geográfica, porte das escolas e período de matrícula dos estudantes (diurno e noturno). A distribuição regional foi observada pelo número de escolas existentes em cada uma das 17 Gerências Regionais de Ensino (GERE). A fim de subsidiar o planejamento amostral, as escolas foram classificadas em três categorias: pequeno porte (menos de 200 alunos); médio porte (200 a 499 alunos); e, grande porte (500 alunos ou mais). Alunos matriculados no período da manhã e da tarde foram agrupados numa única categoria (estudantes do período diurno).

Para seleção da amostra requerida, recorreuse a um procedimento de amostragem aleatória estratificada em dois estágios, e a "escola" e a "turma" representaram as unidades amostrais, respectivamente, no primeiro e no segundo estágio. Todas as escolas da rede pública estadual em Pernambuco foram consideradas elegíveis para inclusão no estudo. No primeiro estágio, adotouse como critério de estratificação para realização do sorteio a densidade de escolas em cada microrregião do estado (GERE) por porte. No segundo estágio, considerou-se a densidade de turmas nas escolas sorteadas por período (diurno e noturno) como critério para sorteio das turmas nas quais os questionários seriam aplicados. Todos os estudantes das turmas sorteadas foram 
convidados a participar do estudo. Os sorteios foram realizados mediante geração de números aleatórios pelo programa Epi Info 6.04d (Centers for Disease Control and Prevention, Atlanta, Estados Unidos).

A coleta dos dados foi realizada no período de abril a outubro de 2006. As medidas antropométricas e a aplicação dos questionários foram efetuadas por seis mestrandos do Programa de Pós-graduação em Hebiatria da Universidade de Pernambuco, que participaram de uma capacitação prévia para padronização dos procedimentos de coleta de dados. A aplicação dos questionários foi efetuada em sala de aula sem a presença dos professores, e todos os estudantes presentes foram convidados a participar, independentemente da idade. Além disso, os questionários não continham qualquer tipo de identificação pessoal (nome, matrícula). Os alunos foram continuamente assistidos pelos aplicadores (sempre dois por turma) para que pudessem esclarecer dúvidas e auxiliar no preenchimento das informações.

O questionário utilizado para obtenção de dados relativos às variáveis independentes foi uma versão traduzida, auto-administrada e previamente testada do Global School-Based Student Health Survey (GSHS), proposto pela Organização Mundial da Saúde (OMS), disponível para consulta na Internet (http://www.who.int/chp/ gshs/en). O questionário é composto por dez módulos: (1) características pessoais; (2) consumo de álcool e drogas; (3) hábitos alimentares; (4) higiene; (5) sentimentos e relacionamentos; (6) atividades físicas; (7) comportamentos na escola; (8) comportamento sexual; (9) tabagismo; e (10) violência.

Previamente ao início da coleta de dados, um estudo piloto foi conduzido a fim de determinar indicadores de reprodutibilidade de medidas e testar a aplicabilidade do instrumento. Os dados para o estudo piloto foram coletados em duas escolas da rede pública municipal de ensino na cidade do Recife, com uma amostra de 138 adolescentes com idade de 14 a 19 anos (59 moças). Indicadores de reprodutibilidade (consistência de medidas teste-reteste) foram de moderados até altos na maioria dos itens do instrumento, e os coeficientes de concordância (índice kappa) variaram de 0,52 a 1,00.

Posteriormente, os estudantes foram encaminhados para outra sala onde se procedeu à coleta das medidas antropométricas. Massa (kg) e estatura $(\mathrm{cm})$ corporal foram verificadas conforme padronização de medidas proposta na literatura especializada 18. Estadiômetros da marca Plenna (modelo 206; São Paulo, Brasil) foram utilizados na determinação da estatura, com uma precisão de 0,5 centímetros (amplitude de medidas de 120 a 220 centímetros), enquanto a medida de massa foi efetuada mediante utilização de balança eletrônica da marca Plenna (modelo Sport), previamente calibrada (amplitude de medidas de $30 \mathrm{~kg}$ a $150 \mathrm{~kg}$ ).

As variáveis dependentes do estudo foram o sobrepeso e obesidade, definidas de acordo com os pontos de corte do índice de massa corporal $\left(\right.$ IMC $=$ massa $/$ altura $\left.^{2}\right)$ propostos pelo International Obesity Task Force (IOTF) e publicados por Cole et al. ${ }^{19}$. A opção por esta referência de avaliação foi decorrente do seu uso mais freqüente em estudos congêneres, permitindo assim eficaz comparação dos achados deste estudo em relação ao que está relatado na literatura especializada. Além disso, para proposição desses pontos de corte, os autores incluíram no estudo uma amostra de adolescentes brasileiros.

As variáveis independentes foram todas auto-referidas e, para fins de análise, foram divididas em: demográficas (sexo, idade e cor da pele/ etnia); sócio-econômicas (morar com os pais, local de residência, escolaridade da mãe e situação ocupacional do adolescente); relacionadas à escola (turno e participação nas aulas de educação física); e comportamentais (prática de atividades físicas; consumo de frutas, verduras e refrigerantes; e tempo de assistência à televisão).

A cor da pele/etnia foi auto-referida e levou em conta seis possíveis respostas (branca, preta, parda, indígena, amarela e outra) e, em seguida, agrupada em duas categorias (branco e não branco). A variável "morar com os pais" foi dicotômica (sim/não) e somente os estudantes que referiram morar com pai e mãe foram classificados na categoria "sim". O local de residência foi referido em duas categorias (urbano e rural). A escolaridade da mãe foi referida pelo filho e classificada em três categorias ( $\leq 8$ anos, $9-11$ anos e 12 ou mais anos de estudo). A situação ocupacional do adolescente foi uma variável dicotômica analisada mediante engajamento do adolescente no mercado de trabalho formal ou informal, assim todos que relataram trabalhar, independentemente de ter ou não carteira profissional assinada, foram classificados como "trabalhadores".

O turno em que estudam foi agrupado em diurno (abrangendo aqueles que estudam no período matutino e vespertino) e noturno. A participação nas aulas de educação física foi estabelecida pela freqüência semanal de assistência às aulas, mas os dados foram agrupados em duas categorias: freqüenta e não freqüenta.

O consumo de frutas, verduras e refrigerantes foi determinado pela análise da freqüência de ingestão nos trinta dias anteriores ao levantamento, considerando-se as seguintes opções de respos- 
ta em relação ao consumo habitual nos últimos trinta dias: não consumi, < 1 vez por dia, 1 vez por dia, 2 vezes por dia, 3 vezes por dia e 4 ou mais vezes por dia. Os adolescentes que relataram um consumo diário de refrigerantes e consumo ocasional ( $<1$ vez por dia) de frutas e verduras foram classificados como expostos a padrão inadequado de consumo desses alimentos.

A freqüência e o tempo de prática de atividade física de intensidade moderada a vigorosa em uma semana típica do adolescente foram considerados a fim de derivar uma medida do nível de atividade física. Os sujeitos que relataram participar de pelo menos 60 minutos de atividades físicas moderadas a vigorosas, durante cinco ou mais dias por semana foram classificados como fisicamente ativos, enquanto os demais foram denominados insuficientemente ativos.

Tempo diante da televisão foi efetuada separadamente para dias de semana e para dias de final de semana, sendo analisadas como duas variáveis independentes. Aqueles que referiram assistir à televisão três ou mais horas por dia foram classificados como "expostos" a excessivo tempo de assistência à televisão. Nas análises bivariada e multivariável, alguns fatores foram dicotomizados: idade (14 a 16/17 a 19 anos), cor da pele (branca/não branca), situação ocupacional do adolescente (trabalha/não trabalha).

O procedimento de tabulação final dos dados foi efetuado pelo programa Epidata (Epidata Association, Odense, Dinamarca), recorrendo-se à entrada dupla e, posteriormente, à comparação dos arquivos de dados gerados para detectar e corrigir erros. Checagens automáticas de amplitude e consistência na entrada de dados também foram adotadas.

A análise foi realizada pelo programa SPSS, versão 10 (SPSS Inc., Chicago, Estados Unidos). Para avaliar a associação entre variáveis, recorreu-se à aplicação do teste de qui-quadrado e, no caso das variáveis em escala ordinal, ao quiquadrado para tendência. Na etapa de análise multivariável, recorreu-se à regressão logística binária, adotando-se a ocorrência do sobrepeso e obesidade como desfechos. Um modelo hierárquico, previamente estabelecido, conforme a recomendação da literatura $20,21,22$, foi adotado para estabelecer a ordem de entrada das variáveis independentes. A abordagem hierárquica utilizada considerou três níveis: (a) distal, no qual foram incluídas as variáveis demográficas (sexo, idade e cor da pele) e sócio-econômicas (morar com os pais, escolaridade materna e situação ocupacional); (b) intermediário, no qual foram incluídos os fatores relacionados à escola (turno e participação nas aulas de educação física); e, (c) proximal, no qual foram incluídos os fatores que expressam o nível de atividade física e o padrão de consumo de frutas, verduras e refrigerantes. No modelo final de regressão foram considerados significativamente associados somente os fatores para os quais o valor de $\mathrm{p}$ foi inferior a 0,05 .

\section{Resultados}

Foram visitadas 76 escolas ( $11 \%$ do total de escolas estaduais do estado) em 44 municípios, o que representa $23 \%$ do total de municípios Pernambucanos. Foram efetivamente entrevistados 4.210 estudantes com idade entre 14 e 19 anos (59,8\% do sexo feminino). Do total de estudantes que estavam presentes nas escolas e turmas sorteadas ( $\mathrm{n}=4.297), 83$ se recusaram a participar ( $1,93 \%$ de recusas). A amostra final $(n=4.210)$, portanto, representa $99,8 \%$ do inicialmente previsto $(\mathrm{n}=4.217$ )

As características demográficas e sócioeconômicas da amostra estão apresentadas na Tabela 1.

A prevalência de sobrepeso e obesidade foi de 11,5\% (IC95\%: 10,7-12,8) e 2,4\% (IC95\%: 1,7$2,7)$, respectivamente. Não se observou diferença significativa entre a proporção de moças e rapazes classificados como casos de "sobrepeso" e de "obesidade". Apesar disso, decidiu-se recorrer à estratificação das análises por sexo tendo em vista que os fatores que podem estar associados à ocorrência desses desfechos podem ser diferentes em rapazes e em moças. Nas análises bivariadas, verificou-se, entre os rapazes, que o local de residência (rural/urbano) foi um fator associado à ocorrência tanto do sobrepeso quanto da obesidade. Encontrou-se uma maior proporção de indivíduos com sobrepeso e obesidade entre aqueles que residem na zona urbana. Constatouse, ainda, que a ocorrência de obesidade estava significativamente associada ao nível de atividade física dos sujeitos, e a prevalência deste evento foi 2,7 vezes maior entre os rapazes classificados como "insuficientemente ativos". Os resultados estão apresentados nas Tabelas 2 e 3.

Entre as moças, as análises bivariadas evidenciaram que a ocorrência de sobrepeso estava associada à faixa etária dos sujeitos $(p=0,05)$ e à participação nas aulas de educação física $(p=0,01)$. A prevalência foi maior entre os sujeitos com idade de 17 a 19 anos em comparação àqueles com idade de 14 a 16 anos. Verificou-se entre os sujeitos que referiram participar das aulas de educação física menor prevalência de sobrepeso. A ocorrência de obesidade entre as moças estava associada ao tempo de exposição à televisão, mas somente em dias de semana, e a prevalência foi 
Tabela 1

Características demográficas e sócio-econômicas da amostra, estratificada por sexo.

\begin{tabular}{|c|c|c|c|c|c|c|}
\hline \multirow[t]{2}{*}{ Variável } & \multicolumn{2}{|c|}{ Rapazes } & \multicolumn{2}{|c|}{ Moças } & \multicolumn{2}{|c|}{ Todos } \\
\hline & $\%$ & $\mathrm{n}$ & $\%$ & $\mathrm{n}$ & $\%$ & $\mathrm{n}$ \\
\hline \multicolumn{7}{|l|}{ Idade (anos) * } \\
\hline 14 & 3,1 & 52 & 5,5 & 137 & 4,5 & 189 \\
\hline 15 & 12,0 & 203 & 17,6 & 443 & 15,4 & 646 \\
\hline 16 & 20,4 & 344 & 23,4 & 587 & 22,2 & 931 \\
\hline 17 & 29,4 & 499 & 24,3 & 615 & 26,4 & 1.114 \\
\hline 18 & 21,7 & 369 & 18,3 & 460 & 19,7 & 829 \\
\hline 19 & 13,4 & 226 & 10,9 & 275 & 11,9 & 501 \\
\hline \multicolumn{7}{|l|}{ Cor da pele/etnia ** } \\
\hline Branco(a) & 24,8 & 419 & 25,5 & 641 & 25,2 & 1.060 \\
\hline Preto(a) & 3,6 & 61 & 1,6 & 40 & 2,4 & 101 \\
\hline Pardo(a)/Mulato(a) & 67,0 & 1.133 & 68,1 & 1.716 & 67,6 & 2.849 \\
\hline Indígena & 0,9 & 15 & 0,6 & 15 & 0,7 & 30 \\
\hline Amarelo(a) & 3,3 & 56 & 4,0 & 101 & 3,7 & 157 \\
\hline Outro & 0,4 & 7 & 0,2 & 6 & 0,3 & 13 \\
\hline \multicolumn{7}{|l|}{ Turno ** } \\
\hline Diurno & 53,9 & 912 & 60,1 & 1.513 & 57,6 & 2.425 \\
\hline Noturno & 46,1 & 780 & 39,9 & 1.005 & 42,4 & 1.785 \\
\hline \multicolumn{7}{|l|}{ Série } \\
\hline $1^{\circ}$ ano & 46,2 & 781 & 43,8 & 1.104 & 44,8 & 1.885 \\
\hline $2^{\circ}$ ano & 30,9 & 524 & 32,6 & 820 & 31,9 & 1.344 \\
\hline $3^{\circ}$ ano & 22,9 & 387 & 23,6 & 594 & 23,3 & 981 \\
\hline \multicolumn{7}{|l|}{ Trabalho ** } \\
\hline Não & 69,3 & 1.167 & 84,8 & 2.140 & 78,6 & 3.306 \\
\hline Sim & 19,1 & 323 & 9,7 & 245 & 13,3 & 568 \\
\hline Estágio & 5,8 & 98 & 3,2 & 82 & 4,3 & 180 \\
\hline Voluntário & 5,8 & 98 & 2,3 & 58 & 3,7 & 156 \\
\hline \multicolumn{7}{|l|}{$\begin{array}{l}\text { Escolaridade materna } \\
\left(\text { anos) }{ }^{\star \star}\right.\end{array}$} \\
\hline$\leq 8$ & 69,4 & 1.255 & 74,5 & 1.781 & 72,5 & 3.046 \\
\hline $9-11$ & 22,5 & 405 & 20,2 & 484 & 21,1 & 889 \\
\hline 12 ou mais & 8,1 & 147 & 5,3 & 128 & 6,4 & 275 \\
\hline \multicolumn{7}{|l|}{ Mora com os pais ** } \\
\hline Sim & 68,1 & 1.147 & 60,6 & 1.534 & 63,6 & 2.681 \\
\hline Não & 31,9 & 536 & 39,4 & 983 & 36,4 & 1.529 \\
\hline
\end{tabular}

${ }^{*} p<0,05$ para teste de qui-quadrado para tendência;

${ }^{\star \star} p<0,05$ para teste de qui-quadrado.

maior entre aquelas que relataram assistir à televisão três ou mais horas por dia. Os resultados estão apresentados nas Tabelas 4 e 5.

Procedeu-se então às analises com ajuste para variáveis de confusão em potencial. Foram incluídas no modelo de regressão multivariável todas as variáveis, independentemente do valor de $\mathrm{p}$ observado nas análises bivariadas. Nos resultados da análise multivariável, evidenciou-se entre os rapazes que tanto para a ocorrência de sobrepeso quanto de obesidade os fatores asso- ciados foram o local de residência e a participação nas aulas de educação física. Mesmo após ajuste para as variáveis potencialmente intervenientes, verificou-se que os rapazes que relataram não freqüentar as aulas de educação física apresentaram maior chance tanto de sobrepeso quanto de obesidade. Em relação ao local de residência, observou-se que os residentes em zonas "urbanas" têm maior chance tanto de sobrepeso quanto de obesidade. 
Tabela 2

Prevalência de sobrepeso (desfecho) entre os rapazes, odds ratio (OR) bruto e ajustado e respectivos intervalos de $95 \%$ de confiança (IC95\%).

\begin{tabular}{|c|c|c|c|c|c|c|c|}
\hline Variáveis * & $\begin{array}{c}\text { Prevalência ** } \\
\%(n)\end{array}$ & OR bruto & IC95\% & Valor de $p$ & OR ajustado *** & IC95\% & Valor de $p$ \\
\hline \multicolumn{8}{|l|}{ Faixa etária (anos) } \\
\hline $14-16$ & $12,4(74)$ & 1,00 & & & 1,00 & & \\
\hline $17-19$ & $10,7(117)$ & 0,85 & $0,65-1,16$ & 0,31 & 0,80 & $0,57-1,14$ & 0,22 \\
\hline \multicolumn{8}{|l|}{ Cor da pele } \\
\hline Branco & $12,9(54)$ & 1,00 & & & 1,00 & & \\
\hline Não branco & $10,9(137)$ & 0,81 & $0,58-1,14$ & 0,24 & 1,23 & $0,86-1,77$ & 0,24 \\
\hline \multicolumn{8}{|l|}{ Local de residência } \\
\hline Rural & $6,5(24)$ & 1,00 & & & 1,00 & & \\
\hline Urbano & $12,7(167)$ & 2,08 & $1,33-3,23$ & 0,01 & 1,79 & $1,07-2,73$ & 0,02 \\
\hline \multicolumn{8}{|l|}{ Trabalho } \\
\hline Não & 12,0 (139) & 1,00 & & & 1,00 & & \\
\hline Sim & $9,7(50)$ & 0,79 & $0,56-1,11$ & 0,17 & 0,71 & $0,48-1,05$ & 0,09 \\
\hline \multicolumn{8}{|l|}{ Mora com a família } \\
\hline $\operatorname{Sim}$ & $10,8(122)$ & 1,00 & & & 1,00 & & \\
\hline Não & $12,1(64)$ & 1,13 & $0,82-1,56$ & 0,43 & 1,58 & $0,75-1,50$ & 0,74 \\
\hline \multicolumn{8}{|l|}{ Escolaridade materna (anos) } \\
\hline$\leq 8$ & $9,9(108)$ & 1,00 & & & 1,00 & & \\
\hline $9-11$ & $14,2(50)$ & 1,32 & $0,98-2,14$ & 0,08 & 1,37 & $0,94-2,00$ & 0,10 \\
\hline 12 ou mais & $15,0(19)$ & 1,48 & $1,01-2,86$ & 0,05 & 1,47 & $0,84-2,56$ & 0,17 \\
\hline \multicolumn{8}{|l|}{ Turno } \\
\hline Diurno & $10,7(97)$ & 1,00 & & & 1,00 & & \\
\hline Noturno & $12,1(94)$ & 1,14 & $0,85-1,55$ & 0,36 & 1,20 & $0,83-1,70$ & 0,33 \\
\hline \multicolumn{8}{|l|}{ Aulas de educação física } \\
\hline Freqüenta & $9,9(66)$ & 1,00 & & & 1,00 & & \\
\hline Não freqüenta & $12,3(125)$ & 1,26 & $0,92-1,73$ & 0,13 & 1,36 & $1,02-1,94$ & 0,04 \\
\hline \multicolumn{8}{|l|}{ Atividade física } \\
\hline Insuficientemente ativo & $11,3(110)$ & 1,00 & & & 1,00 & & \\
\hline Ativo & $11,4(81)$ & 1,00 & $0,74-1,36$ & 0,98 & 1,10 & $0,80-1,53$ & 0,55 \\
\hline \multicolumn{8}{|c|}{$\begin{array}{l}\text { Tempo de assistência à televisão } \\
\text { em dias de semana (horas/dia) }\end{array}$} \\
\hline$<3$ & $11,8(79)$ & 1,00 & & & 1,00 & & \\
\hline$\geq 3$ & $11,0(112)$ & 0,94 & $0,68-1,25$ & 0,61 & 1,02 & $0,71-1,47$ & 0,90 \\
\hline \multicolumn{8}{|c|}{$\begin{array}{l}\text { Tempo de assistência à televisão } \\
\text { em fins de semana (horas) }\end{array}$} \\
\hline$<3$ & $11,6(106)$ & 1,00 & & & 1,00 & & \\
\hline$\geq 3$ & $11,0(85)$ & 0,93 & $0,68-1,26$ & 0,65 & 1,01 & $0,71-1,44$ & 0,92 \\
\hline \multicolumn{8}{|c|}{ Consumo de frutas (vezes/dia) } \\
\hline$<1$ & $10,1(63)$ & 1,00 & & & 1,00 & & \\
\hline$\geq 1$ & $12,0(127)$ & 1,21 & $0,88-1,66$ & 0,20 & 1,26 & $0,87-1,82$ & 0,21 \\
\hline \multicolumn{8}{|c|}{ Consumo de verduras (vezes/dia) } \\
\hline$<1$ & $11,1(70)$ & 1,00 & & & 1,00 & & \\
\hline$\geq 1$ & $11,5(120)$ & 1,03 & $0,75-1,41$ & 0,83 & 1,00 & $0,70-1,43$ & 0,99 \\
\hline \multicolumn{8}{|c|}{ Consumo de refrigerantes (vez/dia) } \\
\hline$<1$ & $12,0(114)$ & 1,00 & & & 1,00 & & \\
\hline$\geq 1$ & $10,6(76)$ & 0,86 & $0,63-1,18$ & 0,36 & 0,96 & $0,69-1,35$ & 0,84 \\
\hline
\end{tabular}

* Fatores demográficos e sócio-econômicos (distal), fatores relacionados à escola (medial) e fatores comportamentais (proximal);

** Teste de razão de verossimilhança para as variáveis binárias e teste de Wald de tendência linear para a variável "escolaridade materna";

*** Todas as variáveis estão controladas para as demais do mesmo nível e para as do nível imediatamente anterior. 
Tabela 3

Prevalência de obesidade (desfecho) entre os rapazes, odds ratio (OR) bruto e ajustado e respectivos intervalos de 95\% de confiança (IC95\%).

\begin{tabular}{|c|c|c|c|c|c|c|c|}
\hline Variáveis * & $\begin{array}{c}\text { Prevalência ** } \\
\%(n)\end{array}$ & OR bruto & IC95\% & Valor de $p$ & OR ajustado *** & IC95\% & Valor de $p$ \\
\hline \multicolumn{8}{|l|}{ Faixa etária (anos) } \\
\hline $14-16$ & $1,7(10)$ & 1,00 & & & 1,00 & & \\
\hline $17-19$ & $2,1(23)$ & 1,26 & $0,60-2,68$ & 0,53 & 1,51 & $0,66-3,45$ & 0,32 \\
\hline \multicolumn{8}{|l|}{ Cor da pele } \\
\hline Branco & $2,6(11)$ & 1,00 & & & 1,00 & & \\
\hline Não branco & $1,7(22)$ & 0,65 & $0,31-1,36$ & 0,25 & 1,33 & $0,59-3,02$ & 0,48 \\
\hline \multicolumn{8}{|l|}{ Local de residência } \\
\hline Rural & $0,5(2)$ & 1,00 & & & 1,00 & & \\
\hline Urbano & $2,4(31)$ & 4,39 & $1,05-18,3$ & 0,04 & 3,36 & $1,48-5,25$ & 0,00 \\
\hline \multicolumn{8}{|l|}{ Trabalho } \\
\hline Não & $2,2(26)$ & 1,00 & & & 1,00 & & \\
\hline Sim & $1,4(7)$ & 0,60 & $0,25-1,39$ & 0,20 & 0,47 & $0,15-1,45$ & 0,19 \\
\hline \multicolumn{8}{|l|}{ Mora com a família } \\
\hline $\operatorname{Sim}$ & $2,0(3)$ & 1,00 & & & 1,00 & & \\
\hline Não & $1,7(9)$ & 0,83 & $0,38-1,81$ & 0,64 & 0,88 & $0,39-2,01$ & 0,77 \\
\hline \multicolumn{8}{|l|}{ Escolaridade materna (anos) } \\
\hline$\leq 8$ & $1,7(18)$ & 1,00 & & & 1,00 & & \\
\hline $9-11$ & $2,6(9)$ & 1,12 & $0,84-2,85$ & 0,41 & 1,45 & $0,62-3,40$ & 0,38 \\
\hline 12 ou mais & $1,6(2)$ & 0,99 & $0,30-2,66$ & 0,53 & 0,85 & $0,18-3,87$ & 0,84 \\
\hline \multicolumn{8}{|l|}{ Turno } \\
\hline Diurno & $2,2(20)$ & 1,00 & & & 1,00 & & \\
\hline Noturno & $1,7(13)$ & 0,75 & $0,37-1,52$ & 0,43 & 0,56 & $0,23-1,34$ & 0,19 \\
\hline \multicolumn{8}{|l|}{ Aulas de educação física } \\
\hline Freqüenta & $1,7(11)$ & 1,00 & & & 1,00 & & \\
\hline Não freqüenta & $2,2(22)$ & 1,31 & $0,63-2,72$ & 0,46 & 1,69 & $1,09-1,88$ & 0,04 \\
\hline \multicolumn{8}{|l|}{ Atividade física } \\
\hline Insuficientemente ativo & $2,7(26)$ & 1,00 & & & 1,00 & & \\
\hline Ativo & $1,0(7)$ & 0,36 & $0,15-0,83$ & 0,01 & 0,49 & $0,20-1,19$ & 0,11 \\
\hline \multicolumn{8}{|c|}{$\begin{array}{l}\text { Tempo de assistência à televisão } \\
\text { em dias de semana (horas/dia) }\end{array}$} \\
\hline$<3$ & $2,1(14)$ & 1,00 & & & 1,00 & & \\
\hline$\geq 3$ & $1,9(19)$ & 0,89 & $0,44-1,78$ & 0,74 & 1,10 & $0,84-1,66$ & 0,36 \\
\hline \multicolumn{8}{|c|}{$\begin{array}{l}\text { Tempo de assistência à televisão } \\
\text { em fins de semana (horas) }\end{array}$} \\
\hline$<3$ & $1,8(16)$ & 1,00 & & & 1,00 & & \\
\hline$\geq 3$ & $2,2(17)$ & 1,25 & $0,62-2,49$ & 0,52 & 1,23 & $0,54-2,80$ & 0,60 \\
\hline \multicolumn{8}{|l|}{$\begin{array}{l}\text { Consumo de frutas } \\
\text { (vezes/dia) }\end{array}$} \\
\hline$<1$ & $1,1(7)$ & 1,00 & & & 1,00 & & \\
\hline$\geq 1$ & $2,5(26)$ & 2,21 & $0,95-5,13$ & 0,06 & 1,90 & $0,99-5,20$ & 0,06 \\
\hline \multicolumn{8}{|c|}{ Consumo de verduras (vezes/dia) } \\
\hline$<1$ & $1,9(12)$ & 1,00 & & & 1,00 & & \\
\hline$\geq 1$ & $2,0(21)$ & 1,05 & $0,51-2,15$ & 0,89 & 0,68 & $0,30-1,53$ & 0,36 \\
\hline \multicolumn{8}{|c|}{ Consumo de refrigerantes (vez/dia) } \\
\hline$<1$ & $2,0(19)$ & 1,00 & & & 1,00 & & \\
\hline$\geq 1$ & $1,9(14)$ & 0,97 & $0,48-1,95$ & 0,94 & 1,35 & $0,62-2,95$ & 0,44 \\
\hline
\end{tabular}

* Fatores demográficos e sócio-econômicos (distal), fatores relacionados à escola (medial) e fatores comportamentais (proximal);

** Teste de razão de verossimilhança para as variáveis binárias e Teste de Wald de tendência linear para a variável "escolaridade materna";

*** Todas as variáveis estão controladas para as demais do mesmo nível e para as do nível imediatamente anterior. 
Tabela 4

Prevalência de sobrepeso (desfecho) entre as moças, odds ratio (OR) bruto e ajustado e respectivos intervalos de $95 \%$ de confiança (IC95\%).

\begin{tabular}{|c|c|c|c|c|c|c|c|}
\hline Variáveis * & $\begin{array}{c}\text { Prevalência ** } \\
\% \text { (n) }\end{array}$ & OR bruto & IC95\% & Valor de $p$ & OR ajustado *** & IC95\% & Valor de $p$ \\
\hline \multicolumn{8}{|l|}{ Faixa etária (anos) } \\
\hline $14-16$ & $10,3(120)$ & 1,00 & & & 1,00 & & \\
\hline $17-19$ & $12,8(172)$ & 1,27 & $1,00-1,63$ & 0,05 & 1,12 & $0,85-1,47$ & 0,4 \\
\hline \multicolumn{8}{|l|}{ Cor da pele } \\
\hline Branco & $10,9(69)$ & 1,00 & & & 1,00 & & \\
\hline Não branco & $12,0(223)$ & 1,12 & $0,84-1,49$ & 0,43 & 0,87 & $0,65-1,19$ & 0,88 \\
\hline \multicolumn{8}{|l|}{ Local de residência } \\
\hline Rural & $11,2(57)$ & 1,00 & & & 1,00 & & \\
\hline Urbano & $11,7(233)$ & 1,05 & $0,77-1,43$ & 0,71 & 1,03 & $0,72-1,43$ & 0,89 \\
\hline \multicolumn{8}{|l|}{ Trabalho } \\
\hline Não & $11,2(238)$ & 1,00 & & & 1,00 & & \\
\hline Sim & $13,6(52)$ & 1,24 & $0,90-1,72$ & 0,17 & 1,21 & $0,85-1,73$ & 0,27 \\
\hline \multicolumn{8}{|l|}{ Mora com a família } \\
\hline Sim & $11,1(167)$ & 1,00 & & & 1,00 & & \\
\hline Não & $12,4(122)$ & 1,14 & $0,89-1,46$ & 0,29 & 1,08 & $0,83-1,41$ & 0,54 \\
\hline \multicolumn{8}{|l|}{ Escolaridade materna (anos) } \\
\hline$\leq 8$ & $11,2(199)$ & 1,00 & & & 1,00 & & \\
\hline $9-11$ & $11,9(57)$ & 1,11 & $0,92-1,56$ & 0,26 & 1,12 & $0,81-1,56$ & 0,46 \\
\hline 12 ou mais & $14,3(18)$ & 1,46 & $1,05-1,99$ & 0,05 & 1,42 & $0,83-2,42$ & 0,19 \\
\hline \multicolumn{8}{|l|}{ Turno } \\
\hline Diurno & $10,8(163)$ & 1,00 & & & 1,00 & & \\
\hline Noturno & $12,9(129)$ & 1,21 & $0,95-1,55$ & 0,11 & 1,14 & $0,86-1,52$ & 0,35 \\
\hline \multicolumn{8}{|l|}{ Aulas de educação física } \\
\hline Freqüenta & $9,3(75)$ & 1,00 & & & 1,00 & & \\
\hline Não freqüenta & $12,8(217)$ & 1,42 & $1,08-1,88$ & 0,01 & 1,24 & $0,91-1,67$ & 0,15 \\
\hline \multicolumn{8}{|l|}{ Atividade física } \\
\hline Insuficientemente ativo & $11,1(195)$ & 1,00 & & & 1,00 & & \\
\hline Ativo & $13,0(97)$ & 1,19 & $0,92-1,55$ & 0,17 & 1,20 & $0,91-1,58$ & 0,19 \\
\hline \multicolumn{8}{|l|}{$\begin{array}{l}\text { Tempo de assistência à } \\
\text { televisão em dias de } \\
\text { semana (horas/dia) }\end{array}$} \\
\hline$<3$ & $12,1(126)$ & 1,00 & & & 1,00 & & \\
\hline$\geq 3$ & $11,4(166)$ & 0,93 & $0,72-1,19$ & 0,57 & 0,87 & $0,66-1,15$ & 0,34 \\
\hline \multicolumn{8}{|l|}{$\begin{array}{l}\text { Tempo de assistência à } \\
\text { televisão em fins de } \\
\text { semana (horas) }\end{array}$} \\
\hline$<3$ & $11,0(129)$ & 1,00 & & & 1,00 & & \\
\hline$\geq 3$ & $12,3(163)$ & 1,14 & $0,89-1,46$ & 0,2 & 1,10 & $0,84-1,45$ & 0,47 \\
\hline \multicolumn{8}{|c|}{ Consumo de frutas (vezes/dia) } \\
\hline$<1$ & $11,9(92)$ & 1,00 & & & 1,00 & & \\
\hline$\geq 1$ & $11,6(200)$ & 0,96 & $0,74-1,25$ & 0,79 & 0,99 & $0,74-1,33$ & 0,95 \\
\hline \multicolumn{8}{|l|}{$\begin{array}{l}\text { Consumo de verduras } \\
\text { (vezes/dia) }\end{array}$} \\
\hline$<1$ & $11,0(98)$ & 1,00 & & & 1,00 & & \\
\hline$\geq 1$ & $12,0(194)$ & 1,13 & $0,85-1,42$ & 0,83 & 1,10 & $0,83-1,45$ & 0,51 \\
\hline \multicolumn{8}{|l|}{$\begin{array}{l}\text { Consumo de refrigerantes } \\
\text { (vezes/dia) }\end{array}$} \\
\hline$<1$ & $11,8(198)$ & 1,00 & & & 1,00 & & \\
\hline$\geq 1$ & $11,1(92)$ & 0,92 & $0,71-1,20$ & 0,58 & 0,84 & $0,64-1,19$ & 0,4 \\
\hline
\end{tabular}

* Fatores demográficos e sócio-econômicos (distal), fatores relacionados à escola (medial) e fatores comportamentais (proximal);

** Teste de razão de verossimilhança para as variáveis binárias e teste de Wald de tendência linear para a variável "escolaridade materna";

*** Todas as variáveis estão controladas para as demais do mesmo nível e para as do nível imediatamente anterior. 
Tabela 5

Prevalência de obesidade (desfecho) entre as moças, odds ratio (OR) bruto e ajustado e respectivos intervalos de $95 \%$ de confiança (IC95\%).

\begin{tabular}{|c|c|c|c|c|c|c|c|}
\hline Variáveis * & $\begin{array}{c}\text { Prevalência } \\
\%(n) * *\end{array}$ & OR bruto & IC95\% & Valor de $p$ & OR ajustado *** & IC95\% & Valor de $p$ \\
\hline \multicolumn{8}{|l|}{ Faixa etária (anos) } \\
\hline $14-16$ & $2,4(28)$ & 1,00 & & & 1,00 & & \\
\hline $17-19$ & $3,1(42)$ & 1,30 & $0,80-2,12$ & 0,16 & 1,23 & $0,73-2,06$ & 0,43 \\
\hline \multicolumn{8}{|l|}{ Cor da pele } \\
\hline Branco & $2,7(17)$ & 1,00 & & & 1,00 & & \\
\hline Não branco & $2,8(53)$ & 1,07 & $0,61-1,86$ & 0,81 & 0,95 & $0,55-1,67$ & 0,87 \\
\hline \multicolumn{8}{|l|}{ Local de residência } \\
\hline Rural & $2,5(13)$ & 1,00 & & & 1,00 & & \\
\hline Urbano & $2,9(57)$ & 1,13 & $0,61-2,08$ & 0,69 & 1,01 & $0,52-1,95$ & 0,97 \\
\hline \multicolumn{8}{|l|}{ Trabalho } \\
\hline Não & $2,7(58)$ & 1,00 & & & 1,00 & & \\
\hline Sim & $3,1(12)$ & 1,15 & $0,61-2,17$ & 0,65 & 1,28 & $0,65-2,50$ & 0,47 \\
\hline \multicolumn{8}{|l|}{ Mora com a família } \\
\hline $\operatorname{Sim}$ & $2,8(42)$ & 1,00 & & & 1,00 & & \\
\hline Não & $2,9(28)$ & 1,02 & $0,63-1,66$ & 0,91 & 0,94 & $0,56-1,57$ & 0,82 \\
\hline \multicolumn{8}{|l|}{ Escolaridade materna (anos) } \\
\hline$\leq 8$ & $2,5(44)$ & 1,00 & & & 1,00 & & \\
\hline $9-11$ & $4,4(21)$ & 1,44 & $1,08-3,33$ & 0,04 & 1,82 & $1,06-3,15$ & 0,03 \\
\hline 12 ou mais & $2,4(3)$ & 0,92 & $0,82-2,95$ & 0,35 & 0,98 & $0,30-3,27$ & 0,98 \\
\hline \multicolumn{8}{|l|}{ Turno } \\
\hline Diurno & $2,7(40)$ & 1,00 & & & 1,00 & & \\
\hline Noturno & $3,0(30)$ & 1,13 & $0,70-1,82$ & 0,61 & 1,14 & $0,66-1,96$ & 0,62 \\
\hline \multicolumn{8}{|l|}{ Aulas de educação física } \\
\hline Freqüenta & $2,4(19)$ & 1,00 & & & 1,00 & & \\
\hline Não freqüenta & $3,0(51)$ & 1,28 & $0,75-2,18$ & 0,35 & 1,15 & $0,65-2,01$ & 0,62 \\
\hline \multicolumn{8}{|l|}{ Atividade física } \\
\hline Insuficientemente ativo & $2,8(49)$ & 1,00 & & & 1,00 & & \\
\hline Ativo & $2,8(21)$ & 1,00 & $0,60-1,69$ & 0,97 & 0,95 & $0,55-1,63$ & 0,85 \\
\hline \multicolumn{8}{|l|}{$\begin{array}{l}\text { Tempo de assistência à } \\
\text { televisão em dias de } \\
\text { semana (horas/dia) }\end{array}$} \\
\hline$<3$ & $2,1(30)$ & 1,00 & & & 1,00 & & \\
\hline$\geq 3$ & $3,8(40)$ & 1,90 & $1,17-3,07$ & 0 & 1,45 & $1,10-2,01$ & 0,04 \\
\hline $\begin{array}{l}\text { Tempo de assistência à } \\
\text { televisão em fins de } \\
\text { semana (horas) }\end{array}$ & & & & & 1,00 & & \\
\hline$<3$ & $2,8(33)$ & 1,00 & & & 1,23 & $0,73-2,06$ & 0,42 \\
\hline$\geq 3$ & $2,8(37)$ & 1,00 & $0,62-1,60$ & 0,99 & & & \\
\hline \multicolumn{8}{|c|}{ Consumo de frutas (vezes/dia) } \\
\hline$<1$ & $2,5(19)$ & 1,00 & & & 1,00 & & \\
\hline$\geq 1$ & $2,9(51)$ & 1,20 & $0,70-2,05$ & 0,49 & 1,23 & $0,69-2,18$ & 0,47 \\
\hline \multicolumn{8}{|l|}{$\begin{array}{l}\text { Consumo de verduras } \\
\text { (vezes/dia) }\end{array}$} \\
\hline$<1$ & $2,4(21)$ & 1,00 & & & 1,00 & & \\
\hline$\geq 1$ & $3,0(49)$ & 1,29 & $0,77-2,17$ & 0,32 & 1,29 & $0,75-2,23$ & 0,35 \\
\hline \multicolumn{8}{|l|}{$\begin{array}{l}\text { Consumo de refrigerantes } \\
\text { (vezes/dia) }\end{array}$} \\
\hline$<1$ & $2,8(47)$ & 1,00 & & & 1,00 & & \\
\hline$\geq 1$ & $2,8(23)$ & 0,98 & $0,57-1,63$ & 0,95 & 1,10 & $0,64-1,67$ & 0,87 \\
\hline
\end{tabular}

* Fatores demográficos e sócio-econômicos (distal), fatores relacionados à escola (medial) e fatores comportamentais (proximal);

** Teste de razão de verossimilhança para as variáveis binárias e teste de Wald de tendência linear para a variável "escolaridade materna";

*** Todas as variáveis estão controladas para as demais do mesmo nível e para as do nível imediatamente anterior. 
Entre as moças, nenhum dos fatores incluídos nas análises estava associado à ocorrência de sobrepeso. O tempo diante da televisão em dias de semana, mas não em dias do final de semana, é um fator associado à obesidade neste grupo. As moças que reportaram assistir à televisão três ou mais horas por dia apresentaram um risco significativamente maior de obesidade.

\section{Discussão}

O presente estudo teve como objetivo analisar a associação entre fatores demográficos, sócioeconômicos, escolares e comportamentais com a prevalência de sobrepeso e obesidade em adolescentes estudantes do Ensino Médio do Estado de Pernambuco.

Os principais resultados foram: a prevalência de sobrepeso e obesidade foi de 11,5\% (IC95\%: 10,7-12,8) e $2,4 \%$ (IC95\%: 1,7-2,7), respectivamente. Após os ajustes para as variáveis de confusão na regressão logística, verificou-se, entre os rapazes, que morar na zona urbana é fator de risco tanto para o sobrepeso $(\mathrm{OR}=1,79$; $\mathrm{IC} 95 \%$ : $1,07-2,73)$ quanto para obesidade $(\mathrm{OR}=3,36$; IC95\%: 1,48-5,25).

Entre as moças, após os ajustes para variáveis de confusão, constatou-se que aquelas que assistem à televisão três horas ou mais em dias de semana apresentam 1,45 (IC95\%: 1,10-2,01) maior risco para obesidade em comparação às que relataram assistir à televisão menos de três horas em dias de semana.

Os resultados indicam que a prevalência tanto de sobrepeso quanto de obesidade neste grupo populacional é inferior ao observado em levantamentos realizados em países europeus 24 e nos países da América do Norte 25,26, mas é similar ao que foi observado em países do leste europeu 27. A similaridade dos resultados observados no presente estudo em relação aos achados de investigações realizadas em países do leste europeu pode ser em decorrência da semelhança das características sócio-econômicas observadas nas populações. Comparando os achados deste estudo com outros levantamentos conduzidos no Brasil, a prevalência de sobrepeso e obesidade foi inferior à observada nas regiões Sul 20,21 e Sudeste 10,28 e semelhante à encontrada em outras investigações realizadas no Nordeste 8,29 . Tais achados são compatíveis com as diferenças regionais já referidas em outros estudos 8,9,10,11.

Nas análises bivariadas não foram encontradas diferenças significativas entre rapazes e moças quanto à prevalência de sobrepeso e de obesidade. Este resultado coincide com os achados relatados por pesquisadores que conduziram estudo na cidade de Pelotas, Rio Grande do Sul, Brasil 20,21 e em São Paulo, Brasil 30. Há, no entanto, estudos que relataram uma maior proporção de obesos entre as moças 8,31 e, ainda, outro que descreveu maior prevalência entre os rapazes 28 . No estudo de Magalhães \& Mendonça ${ }^{9}$, na Região Nordeste a proporção de sujeitos com excesso de peso foi maior entre as moças, enquanto que na Região Sudeste, verificou-se o inverso.

Todavia, os resultados demonstraram também que os fatores associados à ocorrência destes desfechos (sobrepeso e obesidade) são diferentes nos rapazes em comparação ao observado nas moças. No presente estudo, por exemplo, a participação nas aulas de educação física estava associada à ocorrência tanto de sobrepeso quanto de obesidade, mas somente entre os rapazes. Por outro lado, o tempo de assistência à televisão em dias de semana foi identificado como um fator associado à ocorrência somente da obesidade entre as moças. Esses achados reforçam a necessidade de que em estudos congêneres a análise dos fatores associados seja estratificada por sexo, independentemente da existência de diferenças nas prevalências de sobrepeso e ou obesidade.

Em países pobres, questões ligadas à renda, escolaridade da mãe, trabalho e urbanização são determinantes na ocorrência da obesidade juvenil 3. O desenvolvimento econômico nos países em transição favoreceu a urbanização das cidades e êxodo rural, determinando modificações no estilo de vida. Evidências sugerem que pessoas residentes em áreas urbanas apresentam um maior risco de obesidade quando comparados àqueles residentes em áreas rurais 3 . Convergindo para estas evidências, estudos conduzidos nas regiões Nordeste e Sudeste do Brasil indicaram uma maior prevalência de obesidade em residentes na zona urbana em ambas as regiões 8,9.

No presente estudo, após ajuste para fatores de confusão em potencial, constatou-se que os adolescentes do sexo masculino, residentes em área urbana, apresentaram chance $79 \%$ superior de sobrepeso e chance $48 \%$ superior de obesidade em comparação aos residentes em área rural. Uma das possíveis explicações para tal achado é o modo de vida das pessoas residentes em áreas rurais que necessitam fazer outras formas de atividades físicas (deslocamentos, tarefas domésticas pesadas) que não são freqüentes entre as pessoas que residem em áreas urbanas. Outra explicação coerente são os hábitos alimentares dos adolescentes residentes em áreas rurais, onde a disponibilidade de estabelecimentos que comercializam refeições rápidas e o acesso a alimentos industrializados é menor. 
O comportamento alimentar e a prática de atividade física são geralmente apontados como fatores associados à ocorrência de obesidade, mas os resultados de estudos epidemiológicos demonstraram que esta inter-relação não está suficientemente esclarecida 24 e muitos estudos apresentaram resultados divergentes. As principais dificuldades são: (a) avaliar comportamentos complexos e multifatoriais; (b) a maioria dos estudos é feita com medidas auto-relatadas; (c) a maior parte focaliza apenas algumas dimensões dos comportamentos; (d) tais associações são sujeitas ao viés de causalidade reversa e, portanto, estudos prospectivos são necessários para explorar essas relações. Estudos com adolescentes brasileiros que focalizaram a associação entre hábitos alimentares e obesidade são escassos e, em geral, as variáveis de confusão em potencial não foram adequadamente controladas.

No estudo de Andrade et al. 32 , o padrão alimentar de adolescentes cariocas foi investigado, verificando-se numa análise bivariada que os adolescentes com obesidade relataram uma ingestão de gordura total superior em relação aos adolescentes sem sobrepeso. No estudo de Janssen et al. 24, os autores investigaram a associação entre padrões de consumo alimentar e a ocorrência da obesidade em 34 países, verificando-se que o consumo de frutas e verduras não estava associado à obesidade. Os resultados do presente estudo convergem para as evidências apresentadas na literatura quanto à independência entre consumo de frutas e verduras e a ocorrência de sobrepeso e obesidade neste grupo populacional.

Em relação à atividade física, no estudo de Magalhães \& Mendonça 9, observou-se maior proporção de obesos entre as moças que relataram praticar atividades físicas até duas vezes por semana em comparação àquelas mais ativas fisicamente. Similarmente, em estudo realizado com adolescentes (11 a 13 anos) da cidade de Capão da Canoa, Rio Grande do Sul, evidenciou-se associação significativa entre nível de atividade física e ocorrência de sobrepeso 33 . Contrariamente às evidências anteriormente mencionadas, um estudo conduzido em Pelotas encontrou uma prevalência de sobrepeso $42 \%$ superior entre os sujeitos que relataram praticar alguma atividade física fora do ambiente escolar 21. Tais divergências podem ser atribuídas a discrepâncias metodológicas entre os estudos ou ao viés de causalidade reversa, que impede o estudo da relação temporal entre obesidade e atividade física em inquéritos transversais.

Uma tendência recente na literatura tem sido a de considerar nas análises, de modo independente, a prática de atividades físicas e a expo- sição a comportamentos sedentários, especialmente o tempo assistindo à televisão. Estudos transversais $24,33,34$ e ensaios randomizados 35,36 têm evidenciado que tempo de televisão é um importante determinante da obesidade, particularmente entre pessoas jovens.

No Brasil, além de poucos estudos, os resultados são divergentes. No estudo de Terres et al. 21, o tempo diário assistindo à televisão não estava associado à ocorrência de sobrepeso e obesidade. Já no estudo de Sune et al. 33, observou-se uma associação entre a ocorrência de sobrepeso/ obesidade e o tempo diário exposto a comportamentos sedentários (assistir à televisão ou vídeo, jogar videogame e utilizar o computador).

No presente estudo, verificou-se que o tempo de assistência à televisão em dias de semana parece ser um importante determinante da ocorrência de obesidade em adolescentes do sexo feminino, mas a ocorrência desse desfecho foi independente do nível de prática de atividades físicas. Isto sugere que no desenvolvimento de intervenções para controle da obesidade, ações focalizando a redução na exposição a comportamentos sedentários podem constituir importantes e mais efetivas estratégias, sobretudo entre as moças. Trata-se de um achado importante, especialmente ao se considerar que as evidências disponíveis têm sugerido que o tempo de assistência à televisão é uma conduta mais fácil de modificar que a prática de atividades físicas ou alteração de condutas alimentares, além de apresentar forte associação com a redução da obesidade 34,35,36.

A freqüência semanal de participação nas aulas de educação física foi um fator que se manteve associado à ocorrência de obesidade, mesmo após controle das variáveis de confusão em potencial, mas somente entre os rapazes. Foi verificado que não participar das aulas de educação física representa uma chance $45 \%$ superior de obesidade neste grupo. A literatura especializada tem sugerido que as aulas de educação física têm um grande potencial de promoção de conhecimentos e atitudes positivas em relação à saúde 21, entretanto, estudos subseqüentes precisarão explorar melhor tal associação.

Um ponto importante de ser ressaltado é a utilização de diferentes pontos de corte do IMC para determinação da obesidade em adolescentes. O ponto de corte utilizado no presente estudo permitiu a comparação com recentes estudos internacionais 21 e alguns nacionais. Recentemente, com o objetivo de harmonizar as curvas de crescimento e estabelecer novos pontos de corte para as idades de 5 a 19 anos, a OMS estabeleceu a reorganização dos valores de ponto de corte baseados na curva de crescimento de 1977 
e nos valores obtidos em 2007. Um dos aspectos importantes a ser ressaltado é que existe um consenso de que não é possível estabelecer pontos de corte baseados nos diversos levantamentos já realizados em diversos países devido aos diferentes determinantes ambientais e metodologias distintas, o que não permite estabelecer um critério universal 37.

Entre os pontos positivos do presente estudo, podem ser destacados a abrangência do estudo e o tamanho de amostra, suficientes para garantir estimativas de prevalência com razoável precisão e capacidade de detecção de fatores associados com valores de OR superiores a 1,2. Por outro lado, este estudo tem limitações que devem ser consideradas pelos leitores, particularmente o fato de ser um estudo delimitado à população de adolescentes matriculados em escolas do Ensino Médio e somente da rede pública de ensino, aspectos que podem representar um viés de seleção em potencial.

Uma outra limitação foi a impossibilidade de utilizar a renda familiar mensal como indicador do nível sócio-econômico. Isso seria importante porque estudos conduzidos no Brasil 9,10,20,23 evidenciaram que este fator está fortemente associado à ocorrência de obesidade.

Os resultados deste estudo podem auxiliar o planejamento de ações para redução da obesidade em adolescentes. Estratégias que repercutam na redução do tempo diário de assistência à televisão e na maior participação nas aulas de educação física podem ser fatores determinantes para efetividade das intervenções. Futuras investigações poderão explorar meios para promover uma maior participação nas aulas de educação física e reduzir a exposição a comportamentos sedentários em adolescentes.

\section{Resumo}

O objetivo deste estudo foi analisar a associação entre fatores demográficos, sócio-econômicos, escolares e comportamentais e a prevalência de sobrepeso e obesidade em adolescentes. A amostra foi constituída por 4.210 estudantes (14-19 anos) da rede pública estadual em Pernambuco, Brasil, selecionados mediante amostragem por conglomerados em dois estágios. Além das medidas antropométricas, dados pessoais e comportamentais foram coletadas usando o Global School-Based Health Survey. Pontos de corte utilizados para classificação dos casos de sobrepeso e obesidade foram os propostos pelo International Obesity Task
Force. A prevalência de sobrepeso e obesidade foi 11,5\% (IC95\%: 10,7-12,8) e 2,4\% (IC95\%: 1,9-2,9), respectivamente. Verificou-se maior risco de sobrepeso e obesidade entre rapazes que residiam em áreas urbanas e que não participavam das aulas de educação física. Entre as moças, assistir à televisão três horas ou mais foi fator associado à ocorrência de obesidade. Redução do tempo de televisão e participação nas aulas de educação física podem ser ações efetivas no enfrentamento da epidemia da obesidade.

Obesidade; Sobrepeso; Saúde do Adolescente 


\section{Colaboradores}

R. M. Tassitano e M. V. G. Barros lideraram o delineamento do estudo, a redação do manuscrito e as análises estatísticas. R. M. Tassitano, M. C. M. Tenório, J. Bezerra efetuaram a revisão de literatura, a coleta e a tabulação dos dados. P. C. Hallal colaborou revisando criticamente o manuscrito, além de cooperar com as análises estatísticas. Todos os autores colaboraram com a revisão crítica e aprovaram a versão final do manuscrito.

\section{Agradecimentos}

À Secretaria de Educação e Cultura de Pernambuco e às Gerências Regionais de Educação pelo apoio logístico. Aos diretores e professores das escolas que contribuíram para a execução da coleta dos dados. Aos integrantes do Grupo de Pesquisa em Estilos de Vida e Saúde da Escola Superior de Educação Física da Universidade de Pernambuco que colaboraram na coleta e tabulação dos dados. Ao Conselho Nacional de Desenvolvimento Científico e Tecnológico (CNPq; Apoio a Projetos de Pesquisa - processo 486023/2006-0) pelo apoio financeiro.

\section{Referências}

1. World Health Organization. Obesity: preventing and managing the global epidemic. Geneva: World Health Organization; 2000. (Technical Report Series, 894).

2. Reilly JJ. Descriptive epidemiology and health consequences of childhood obesity. Best Pract Res Clin Endocrinol Metab 2005; 19:327-41.

3. Reilly JJ. Tracking the obesity epidemic: new approaches. Arch Dis Child 2006; 91:724-6.

4. Wang Y. Cross-national comparison of childhood obesity: the epidemic and the relationship between obesity and socioeconomic status. Int J Epidemiol 2001; 30:1129-36.

5. Morabia A, Costanza MC. The obesity epidemic as harbinger of a metabolic disorder epidemic: trends in overweight, hypercholesterolemia, and diabetes treatment, in Geneva, Switzerland, 1993-2003. Am J Public Health 2005; 95:632-5.
6. Wang Y, Monteiro C, Popkin BM. Trends of obesity and underweight in older children and adolescents in the United States, Brazil, China, and Russia. Am J Clin Nutr 2002; 75:971-7.

7. Kain J, Vio F, Albala C. Obesity trends and determinant factors in Latin America. Cad Saúde Pública 2003; 19 Suppl 1:S77-86.

8. Abrantes MM, Lamounier JA, Colosimo EA. Prevalência de sobrepeso e obesidade em crianças e adolescentes das regiões Sudeste e Nordeste. J Pediatr (Rio J.) 2002; 78:335-40.

9. Magalhães VC, Mendonça GAS. Prevalência e fatores associados a sobrepeso e obesidade em adolescentes de 15 a 19 anos das regiões Nordeste e Sudeste do Brasil, 1996 a 1997. Cad Saúde Pública 2003; 19 Suppl 1:S129-39. 
10. Neutzeling MB, Taddei JA, Rodrigues EM, Sigulem DM. Overweigh and obesity in brazilian adolescents. Int J Obes Relat Metab Disord 2000; 24 869-74.

11. Veiga GV, Cunha AS, Sichieri R. Trends in overweight among adolescents living in the poorest and richest regions of Brazil. Am J Public Health 2004; 94:1544-8.

12. Vasconcelos VL, Silva GAP. Prevalência de sobrepeso e obesidade em adolescentes masculinos, no Nordeste do Brasil, 1980-2000. Cad Saúde Pública 2003; 19:1445-51.

13. Alves JGB, Siqueira PP, Figueiroa JN. Overweight and physical activity in children living in favelas in the metropolitan region of Recife, Brazil. J Pediatr (Rio J.) 2009; 85:67-71.

14. Silva GAP, Balaban G, Nascimento EMM, Baracho JDS, Freitas MMV. Prevalência de sobrepeso e obesidade em adolescentes de uma escola da rede pública do Recife. Rev Bras Saúde Matern Infant 2002; 2:37-42.

15. Balaban G, Silva GAP. Prevalência de sobrepeso e obesidade em crianças e adolescentes de uma escola da rede privada de Recife. J Pediatr (Rio J.) 2001; 77:96-100.

16. Balaban G, Silva GAP, Motta MEFA. Prevalência de sobrepeso e obesidade em escolares de diferentes classes socioeconômicas em Recife, PE. Pediatria (São Paulo) 2001; 23:285-9.

17. Hebebrand J, Hinney A. Environmental and genetic risk factors in obesity. Child Adolesc Psychiatr Clin N Am 2009; 18:83-94.

18. Lohman TG, Roche AF, Martorell R. Anthropometric standardization reference manual. Champaign: Human Kinetics Books; 1991.

19. Cole TJ, Bellizzi MC, Flegal KM, Dietz WH. Establishing a standard definition for child overweight and obesity worldwide: international survey. BM] 2000; 320:1240-3.

20. Dutra CL, Araújo CL, Bertoldi AD. Prevalência de sobrepeso em adolescentes: um estudo de base populacional em uma cidade no Sul do Brasil. Cad Saúde Pública 2006; 22:151-62.

21. Terres NG, Pinheiro RT, Horta BL, Pinheiro KAT, Horta LL. Prevalência e fatores associados ao sobrepeso e à obesidade em adolescentes. Rev Saúde Pública 2006; 40:627-33.

22. Victora CG, Huttly SR, Fuchs SC, Olinto MT. The role of conceptual frameworks in epidemiological analysis hierarchical approach. Int J Epidemiol 1997; 26:224-7.

23. Monteiro P, Victora C, Barros F. Fatores de risco sociais, familiares e comportamentais para obesidade em adolescentes. Rev Panam Salud Pública 2004; 16:250-8.
24. Janssen I, Katzmarzyk PT, Boyce WF, Vereecken C, Mulvihill C, Roberts C, et al. Comparison of overweight and obesity prevalence in school-aged youth from 34 countries and their relationship with physical activity and dietary patterns. Obes Rev 2005; 6:123-32.

25. Odgen CL, Carroll MD, Curtin LR, McDowell MA, Tabak CJ, Flegal KM. Prevalence of overweight and obesity in the united States, 1999-2004. JAMA 2006; 295:1549-55.

26. Tremblay MS, Katzmaryk PT, Willms JD. Temporal trends in overweight and obesity in Canada, 1981-1996. Int J Obes Relat Metab Disord 2002; 26: 538-43.

27. Lobstein T, Frelut ML. Prevalence of overweight among children in Europe. Obes Rev 2003; 4: 195-200.

28. Ramos AMPP, Barros Filho AA. Prevalência da obesidade em adolescentes de Bragança Paulista e sua relação com a obesidade dos pais. Arq Bras Endocrinol Metab 2003; 47:663-8.

29. Silva MAM, Rivera IV, Ferraz RMT, Pinheiro AJT, Alves SWS, Moura AA, et al. Prevalência de fatores de risco cardiovascular em crianças e adolescentes da rede de ensino da cidade de Maceió. Arq Bras Cardiol 2005; 84:387-92.

30. Nobre MRC, Domingues RZZL, Silva AR, Colugnati FAB, Taddei JAC. Prevalências de sobrepeso, obesidade e hábitos de vida associados ao risco cardiovascular em alunos do ensino fundamental. Rev Assoc Med Bras 2006; 52:118-24.

31. Florêncio TM, Ferreira HS, Franca AP, Cavalcante JC, Sawava AL. Obsesity and undernutrition in very low-income population in the city of Maceió, northeast Brazil. Br J Nutr 2001; 86:277-83.

32. Andrade RG, Pereira RA, Sichieri R. Consumo alimentar de adolescentes com e sem sobrepeso do Município do Rio de Janeiro. Cad Saúde Pública 2003; 19:1485-95.

33. Suñé FR, Dias-da-Costa JS, Olinto MTA, Pattussi MP. Prevalência e fatores associados para sobrepeso e obesidade em escolares de uma cidade no Sul do Brasil. Cad Saúde Pública 2007; 23:1361-71.

34. Patrick K, Norman GJ, Calfas KJ, Sallis JF, Zabinski MF, Rupp J, et al. Diet, physical activity, and sedentary behaviors as risk factors for overweight in adolescence. Arch Pediatr Adolesc Med 2004; 158:385-90.

35. Robinson TN. Reducing children's television viewing to prevent obesity: a randomizer controlled trial. JAMA 1999; 282:1561-7.

36. Sharma M. School-based interventions for childhood and adolescent obesity. Obes Rev 2006; 7:261-9.

37. Onis M, Onyango AW, Borghi E, Siyam A, Nishida C, Siekmann J. Development of a WHO growth reference for school-aged children and adolescents. Bull World Health Organ 2007; 85:660-7.

Recebido em 08/Set/2008

Versão final reapresentada em 10/Set/2009

Aprovado em 14/Set/2009 